\title{
In-situ marine gas hydrate production methane leaks electrical monitoring system
}

\author{
Hanfu $\mathrm{Wu}^{1}$, Xiujun Guo ${ }^{1,2, a}$, Jingxin $\mathrm{Wu}^{1}$, and Yufeng Zhang ${ }^{1}$ \\ ${ }^{1}$ Ocean University of China, College of Environmental Science and Engineering, 266100 Qingdao, China \\ ${ }^{2}$ Key Laboratory of Marine Environment and Geological Engineering of Shandong Province , 266100 Qingdao, China
}

\begin{abstract}
In the process of gas hydrate exploitation, methane leakage needs to be monitored in real time, so an in-situ electrical monitoring system for methane leakage is designed. The monitoring system is mainly composed of monitoring cable, acquisition station, power module and general control platform. According to the electrical principle, the system carries out regional monitoring on the seabed formation, forms the resistivity map, and realizes methane leakage monitoring. The cost of the monitoring system is low, and it can be remotely controlled or automatically collected data according to the preset program, so the system has good application and research value.
\end{abstract}

\section{Introduction}

Natural gas hydrate is considered to be the most potential energy in the 21 st century ${ }^{[1]}$, but the development of natural gas hydrate will face the risks of methane leakage, seabed subsidence, submarine landslide, pockmarks and so on ${ }^{[2-7]}$. In order to ensure the safety of the project, it is necessary to monitor the leakage of methane gas in real time in the process of natural gas hydrate exploitation. At present, the mainstream monitoring methods are the bottom mounted submarine buoy integrated with methane concentration sensor and horizontal multi beam sonar device, as well as the air trap device ${ }^{[8-9]}$. The former can monitor methane concentration in water in real time, while the latter can monitor gas leakage rate. In addition, Deepsea Seismic System can also be used to retrieve methane leakage from seismic profiles of sedimentary layers in different periods ${ }^{[10]}$. However, there are some limitations in the above methods, the monitoring range of methane leakage monitoring technology of submarine buoy is small and has a certain lag, the operation cost of DSS is high, and it takes a lot of labour and material resources to obtain the monitoring data each time.

Methane gas and hydrate are both non-conductive media in pore space, so marine resistivity method also has the application premise for methane leakage monitoring.
Shang et al (2016) initially constructed the resistivity model of gas bearing sand, and obtained the resistivity imaging profile of gas bearing soil layer through theoretical simulation and indoor experiments, which confirmed the effectiveness of resistivity imaging technology for gas bearing layer detection ${ }^{[11]}$. Breen et al. (2012) studied the ability of electrical resistivity tomography (ERT) to describe the spatial distribution of shallow gas and to estimate gas saturation by using twodimensional model experiments ${ }^{[12]}$. Resistivity imaging method has many practical applications in monitoring gas storage and migration, gas content in pore space ${ }^{[13-15]}$. Both model and in-situ experiments show that resistivity imaging technology can effectively describe the spatial distribution of non-conductive medium in pore space and preliminarily estimate its saturation. Based on above method, we uses the resistivity acquisition technology to design an in-situ electrical monitoring system to monitor methane leakage.

\section{Structure Design of Monitoring System}

Methane leakage in situ electrical monitoring system is mainly composed of four parts: monitoring cable, acquisition station, power module and master control platform . The monitoring cable is connected with the

\footnotetext{
a guojunqd@ouc.edu.cn
} 


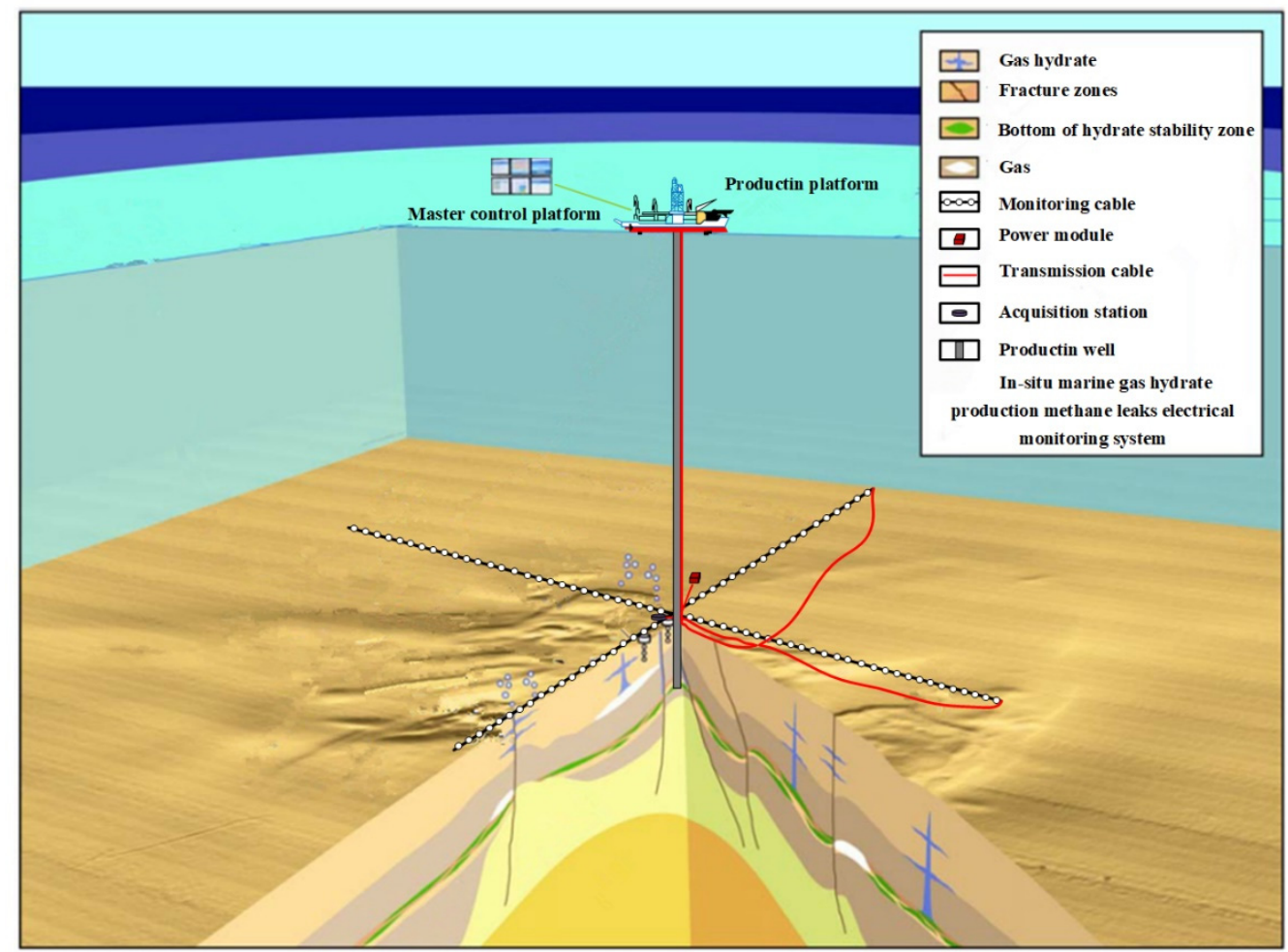

Figure 1. In-situ marine gas hydrate production methane leaks electrical monitoring system

acquisition station, which can be set as single line twodimensional monitoring or double line cross threedimensional monitoring. The monitoring cable is equidistant with multiple electrodes, and each electrode is connected with an electrode conversion module. The acquisition station and power module are located near the production well. The master control platform is located in the production platform, which generates the control instructions for controlling the working state of the acquisition station and sends them to the acquisition station. At the same time, the collected data is sent to the master control platform through the transmission cable. The master control platform calculates the apparent resistivity distribution of the seabed section in the layer, and carries out inversion monitoring. The system structure is shown in Figure 1.

\section{Components of Monitoring System}

\subsection{Monitoring Cable}

The monitoring cable is laid horizontally and symmetrically on both sides with the production well as the center, which can monitor the potential data of the submarine sediment section with the production well as the center and the monitoring cable as the diameter, that is, the key monitoring area in the production process.

The monitoring cable is connected with the acquisition station and is provided with a plurality of equidistant electrodes and corresponding electrode conversion modules. The electrode conversion module controls the working state of the electrodes, and the electrode conversion modules are connected through the internal wires of the monitoring cable. The material of the electrode is high voltage resistance, corrosion resistance and good conductivity, such as titanium steel. Generally speaking, the smaller the electrode distance is, the higher the resolution is, but the cost is also high.

As shown in Figure 2, the electrode conversion module comprises the following parts connected in turn: The interface unit is used to receive the instruction code sent by the acquisition station; The decoding unit decodes the instruction code received by the interface unit and outputs the processing result; The instruction detection unit for detecting and outputting a control instruction from the decoding unit; The control driving unit receives the control command output by the command detection unit and controls the working state of the electrode connected with the electrode conversion module.

\subsection{Acquisition Station}

The acquisition station receives the control instructions from the master control platform to work. The acquisition station has a high-precision clock, and it can also work according to the preset program when it is separated from the master control platform. The specific working mode of the acquisition station is as follows: by controlling the electrode conversion module, the working state of the electrode on the monitoring cable is controlled, and the power supply electrode and the measuring electrode are selected; For the power supply electrode, the control power module of the acquisition station supplies power for it. For the measuring electrode, the acquisition station receives the current potential data collected by it; The acquisition station sends the measured potential data of each layer to the master control platform.

The acquisition station includes the following circuit units (Figure 2): 
The main processing unit is used to generate the

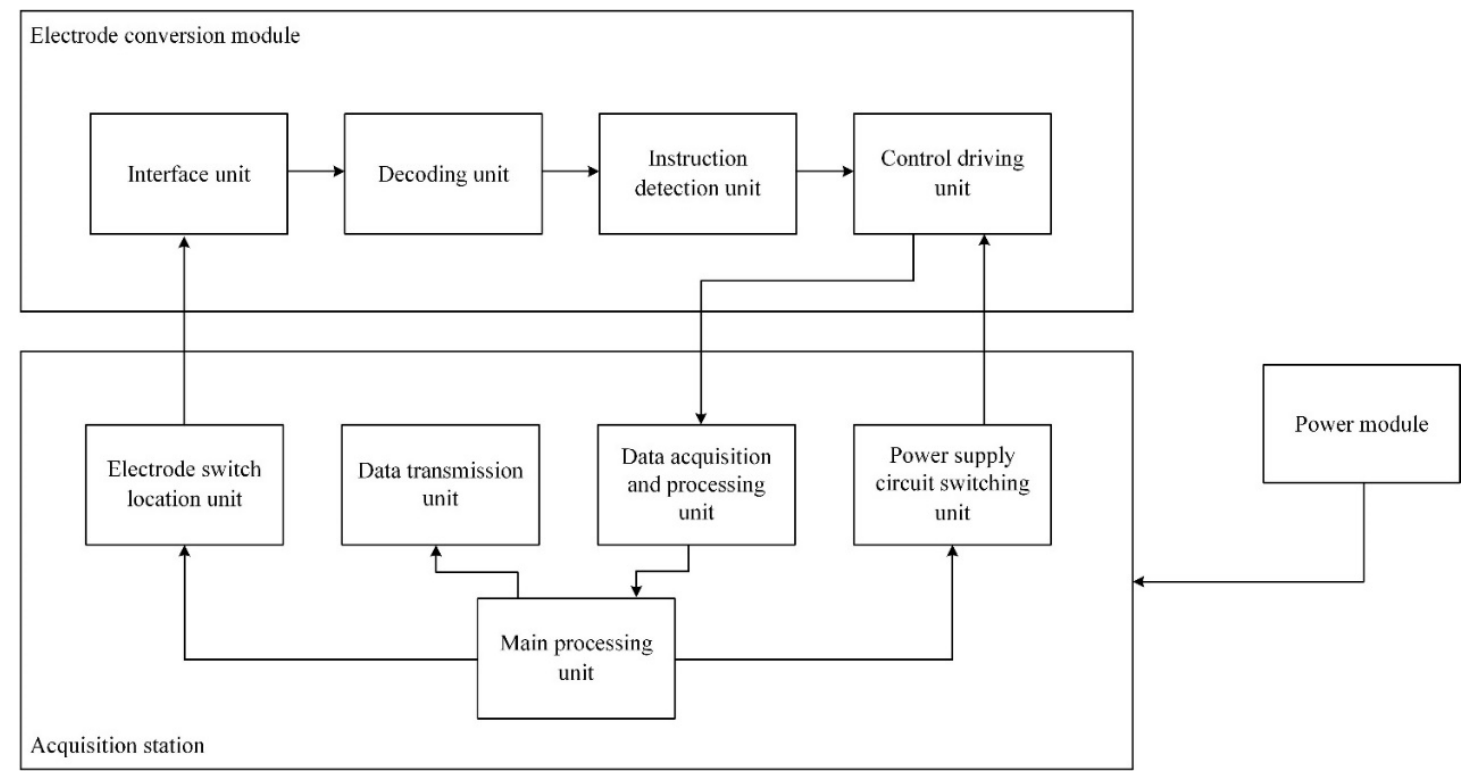

Figure 2. Circuit diagram of electrode conversion module and acquisition station

control signal to control the working state of the electrode, and receive the potential data sent by the electrode conversion module. It can receive the instructions of the main control platform to work, and can also work independently according to the preset schedule without the main control platform through the preset program;

An electrode switch location unit, which is connected with the main processing unit and sends instructions to encode to the interface unit of the electrode conversion module under the control of the main processing unit;

The power supply circuit switching unit is connected with the control driving unit of the electrode conversion module on the one hand and the power supply module on the other hand, and determines whether to supply power for the power supply circuit of the electrode conversion module under the control of the main processing unit;

The data acquisition and processing unit is connected with the control driving unit of the electrode conversion module for collecting and processing current and potential data.

\section{Basic Principles of Monitoring System}

Because the conductivity of the seabed sediments is relatively stable, the methane produced by the decomposition of natural gas hydrate enters into the pores of the seabed sediments in a gaseous manner, and the change of methane content in the seabed sediments directly leads to the change of the resistivity of the seabed sediments. Therefore, this method can indirectly monitor the methane content in seabed sediments by monitoring the resistivity of seabed sediments. Based on the traditional high-density resistivity method, the monitoring cable is laid on the seabed to monitor the apparent resistivity of seabed sediments.

\section{Working Method of Monitoring System}

The acquisition station takes one end of the monitoring cable as the starting end, and selects four electrodes as the working electrodes. Two working electrodes are power supply electrode A and power supply electrode B, one is applied with positive voltage, the other is applied with negative voltage, and the other two working electrodes are measuring electrode $\mathrm{M}$ and measuring electrode $\mathrm{N}$ to measure the electrode position potential data. The control power module of the acquisition station supplies power to the power supply electrode A and the power supply electrode B to form a current loop, and controls the measuring electrode $\mathrm{M}$ and the measuring electrode $\mathrm{N}$ to return their current potential data to complete a single data point acquisition. The power supply electrode $A$, the power supply electrode $B$, the measuring electrode $M$ and the measuring electrode $\mathrm{N}$ move one or more electrode distances to the other end of the monitoring cable at the same time, The next data point is collected until the working electrode moves to the other end of the monitoring cable to complete the measurement of the potential data of one layer on the seabed section. The distance between the layer and the seabed should be given in combination with the geological background of the seabed sedimentary layer in practical application. After measuring the potential data of one layer on the seabed section, increase the distance between the power supply electrode and the measuring electrode by one or more electrode distances, take any end of the monitoring cable as the starting end, select four electrodes as the working electrode, and make the current selected distance between the power supply electrode and the measuring electrode meet the re determined value, and repeat the measurement steps, In this way, the layer by layer measurement is continuously rolled until all the layer potential data of the preset seabed section are measured. 
The acquisition station sends the measured potential data and corresponding current data of each layer of the seabed section to the master control platform, and the platform calculates the apparent resistivity distribution of the seabed section in this layer. The master control platform continues to invert the obtained apparent resistivity data into resistivity data through professional inversion software, and draws the output resistivity profile of the resistivity of each layer of the seabed section. By using the corresponding relationship between the resistivity of the seabed section and the pore water gas saturation of the seabed sedimentary layer, the methane leakage monitoring of the seabed sedimentary layer is realized according to the resistivity change of the seabed section. When there is abnormal high resistance in the overlying soil layer of the seabed sedimentary layer, it is judged as methane leakage and an alarm is given.

In the process of natural gas hydrate exploitation, the above measurement process is carried out regularly to obtain the apparent resistivity profiles of seabed sediments in different periods and monitor the methane leakage range in different periods.

\section{Summary}

The In-situ marine gas hydrate production methane leaks electrical monitoring system is mainly composed of monitoring cable, acquisition station, power module and master control platform. According to the principle of electricity, the system can monitor the area of the seabed sediment, realize the resistivity imaging measurement within a certain range of sedimentary layer with the production well as the center, form the resistivity image, and realize the two-dimensional / three-dimensional monitoring of methane leakage. The system only needs to lay the monitoring cable once, and then it can be controlled remotely or collected automatically according to the setting program. Moreover, the system only needs the power module to provide power, so the operation cost is low. The horizontal monitoring range of the system is usually more than $500 \mathrm{~m}$, and the monitoring is carried out for the sedimentary layer, with strong early warning.

\section{Acknowledgement}

This research was financially supported by the National Natural Science Foundation.of China (41427803).

\section{References}

1. Beaudoin Y C,Boswell R, Dallimore S R, et al. Frozen Heat -A Global Outlook on Methane Gas Hydrates. Norway: Birkeland Trykkeri A/S, 1-29,(2014)

2. Li X S, Xu C G, Zhang Y, et al. Investigation into gas production from natural gas hydrate: A review. Applied Energy, 172:286-322(2016)

3. Paull C K, Dillon W P. Natural Gas Hydrates: Occurrence, Distribution, and Detection. Handbook of Hydrocarbon \& Lipid Microbiology, 1(522):413434(2001)
4. Yokoyama T, Nakatsuka Y. Monitoring System of Seafloor Deformation for Methane Hydrate Production Test. The Japanese Geotechnical Society, 63:26-29 (2015)

5. Stenvold T, Eiken O, Zumberge M, et al. HighPrecision Relative Depth and Subsidence Mapping from Seafloor Water Pressure Measurements. Spe Journal, 11(3):380-389 (2006)

6. Hall S A, Macbeth C, Barkved O I, et al. Time-lapse seismic monitoring of compaction and subsidence at Valhall through cross-matching and interpreted warping of 3D streamer and OBC data. Seg Technical Program Expanded Abstracts, 21(1):1696 (2002)

7. Higley P, Woolsey J R, Goodman R, et al. support of gulf of mexico hydrate research consortium: activities to support establishment of a sea floor monitoring station project. Office of Scientific \& Technical Information Technical Reports (2005)

8. Nagakubo S, Arata N, Yabe I, et al. Environmental impact assessment study on Japan's methane hydrate R\&D program. Fire Ice, 11: 4-11(2011)

9. Waarum I K, Sparrevik P, Kvistedal Y, et al. Innovative Methods for Methane Leakage Monitoring Near Oil and Gas Installations. Offshore Technology Conference (2016)

10. Takahashi H, Asakawa E, Hayashi T, et al. Development of Ocean Bottom Multi-component Seismic System for Methane Hydrate Dissociation Monitoring. American Geophysical Union (2011)

11. Shang K, Guo X, Wu J, Jia Y. Detecting cold spring gas leakage in seabed sediment with marine multielectrode resistivity method:numerical simulation and experiment. Haiyang Xuebao, 039(011): 85-96 (2017)

12. Breen S J, Carrigan C R, LaBrecque D J, et al. Benchscale experiments to evaluate electrical resistivity tomography as a monitoring tool for geologic $\mathrm{CO} 2$ sequestration. International Journal of Greenhouse Gas Control, 9: 484-494 (2012)

13. Terry N, Slater L, Comas X, et al. Free phase gas processes in a northern peatland inferred from autonomous field - scale resistivity imaging. Water Resources Research, 52(4) (2016)

14. Schütze C, Sauer U, Beyer K, et al. Natural analogues: a potential approach for developing reliable monitoring methods to understand subsurface $\mathrm{CO} 2$ migration processes. Environmental Earth Sciences, 67(2): 411-423 (2012)

15. Mendonça C A, Doherty R, Amaral N D, et al. Resistivity and induced polarization monitoring of biogas combined with microbial ecology at a brownfield site. 3(4):SAB43-SAB56 (2015) 